ARQGA/1220

\title{
RELAÇÃO ENTRE IDADE E MOTILIDADE DO ESÔFAGO EM PACIENTES COM DOENÇA DO REFLUXO GASTROESOFÁGICO
}

\author{
Roberto Oliveira DANTAS e Lilian Rose Otoboni APRILE
}

RESUMO - Racional - O comprometimento das contrações esofágicas pode agravar as lesões em esôfago conseqüentes ao refluxo gastroesofágico. A hipótese do presente estudo é de que alterações das contrações são limitadas ao esôfago distal e pioram com o processo de envelhecimento. Objetivo - Avaliar as contrações esofágicas em parte proximal e distal do esôfago em pacientes com sintomas de refluxo gastroesofágico, com e sem lesões no esôfago. Casuística e Métodos - Estudaramse pelo método manométrico as contrações esofágicas a 2, 7, 12 e $17 \mathrm{~cm}$ do esfíncter superior do esôfago, após cinco deglutições de $5 \mathrm{~mL}$ de água, em 104 pacientes com pirose e regurgitação ácida, 42 com endoscopia do esôfago normal, 47 com esofagite moderada e 15 com esofagite grave. Resultados - Nas medidas realizadas a 12 e 17 cm do esfíncter superior do esôfago, a amplitude e área sob a curva das contrações esofágicas foram menores nos pacientes com esofagite grave, quando comparadas com os pacientes sem esofagite ou com esofagite moderada. A 2 e $7 \mathrm{~cm}$ do esfíncter superior do esôfago não houve diferenças na amplitude e área sob a curva das contrações. Não houve diferenças entre os grupos, em todo o esôfago, em relação à duração das contrações, velocidade de propagação do peristaltismo e nas proporções de contrações falhas, simultâneas, não propagadas e peristálticas. Não houve diferenças entre os pacientes com mais de 50 anos e aqueles com menos de 50 anos. Conclusões - Esofagite grave esteve associada com diminuição da amplitude das contrações esofágicas, alteração limitada à parte distal do esôfago. Não foi observado efeito do envelhecimento nas contrações.

DESCRITORES - Refluxo gastroesofágico. Esofagite péptica. Transtornos da motilidade esofágica. Envelhecimento.

\section{INTRODUÇÃO}

A doença do refluxo gastroesofágico (DRGE) é conseqüência da exposição prolongada da mucosa esofágica ao conteúdo gástrico ${ }^{(22)}$, da baixa resistência da mucosa à agressão pelo refluído e da agressividade deste material.

A duração da exposição está relacionada com o número de episódios de refluxo, da capacidade do esôfago em retirar o volume refluído, devolvendo-o ao estômago, e da neutralização do seu componente ácido com a chegada de saliva ao esôfago distal ${ }^{(11)}$.

A retirada do material refluído do esôfago é dependente da motilidade do órgão ${ }^{(13)}$. Contrações não propagadas, de baixa amplitude ou simultâneas, o que define a motilidade do esôfago ineficaz ${ }^{(15,16)}$, comprometem o mecanismo de depuração esofágica ${ }^{(22)}$.
O objetivo deste trabalho foi estudar a motilidade do corpo do esôfago em pacientes com sintomas de refluxo gastroesofágico com e sem esofagite, com a hipótese de que pacientes com esofagite mais grave têm alterações na motilidade do esôfago mais intensas do que aqueles sem esofagite. Examinou-se, também, a possibilidade daqueles com mais idade terem mais alterações do que os com menos idade, considerando que há resultados que mostram que a amplitude da contração esofágica e a proporção de contrações peristálticas diminuem com o envelhecimento ${ }^{(7)}$ e a hipótese de que as possíveis alterações da contração estejam restritas à parte distal do esôfago, uma vez que em trabalho anterior observouse que as contrações em parte proximal do esôfago de pacientes com sintomas de DRGE são semelhantes às de controles ${ }^{(4)}$.

Departamento de Clínica Médica - Faculdade de Medicina de Ribeirão Preto da Universidade de São Paulo - Ribeirão Preto, SP.

Endereço para correspondência: Dr. Roberto Oliveira Dantas - Departamento de Clínica Médica - Faculdade de Medicina de Ribeirão Preto - USP - 14049-900 - Ribeirão Preto, SP. e-mail: rodantas@fmrp.usp.br 


\section{MATERIAL E MÉTODO}

Em período de 2 anos realizou-se manometria do esôfago em 104 pacientes com queixas de pirose e regurgitação ácida. O exame foi parte da investigação diagnóstica de DRGE, que incluía também exames radiológico e endoscópico do esôfago. Neste grupo de pacientes o exame radiológico foi normal.

A endoscopia foi normal em 42 pacientes ( 10 homens), com idades entre 17 e 78 anos (mediana 41 anos), revelou esofagite moderada em 47 (17 homens), com idades entre 19 e 73 anos (mediana 46 anos), e esofagite grave em 15 ( 8 homens), com idades entre 36 e 81 anos (mediana 50 anos). Os pacientes com endoscopia normal não apresentavam qualquer lesão em mucosa do esôfago, aqueles com esofagite moderada tinham lesões que correspondiam aos graus I e II da classificação de Savary-Miller ${ }^{(21)}$, e com esofagite grave aos graus III e IV desta classificação, sendo que seis pacientes tinham esôfago de Barrett. Os pacientes referiam melhora dos sintomas, em diferentes graus de intensidade, com o tratamento clínico com inibidor da bomba de prótons.

$\mathrm{Na}$ avaliação da motilidade esofágica utilizou-se o método manométrico, com um cateter de polivinil de oito canais com diâmetro externo de 4,5 mm e diâmetro interno em cada canal de $0,8 \mathrm{~mm}$ (Arndorfer Specialties, Inc, Greendale, Wisconsin, EUA). Os quatro canais proximais tinham aberturas laterais distanciadas de $5 \mathrm{~cm}$, formando ângulos de $90^{\circ}$. Foram conectados a transdutores de pressão (pub Medizintechnick Gmb H, Kirchseeon, Alemanha), ligados ao aparelho PC Polygraph HR (Synectics Medical, Stockholm, Suécia). Os sinais manométricos foram arquivados em computador. Durante o exame, o cateter foi perfundido continuamente com água a um fluxo de $0,5 \mathrm{~mL} /$ minuto em cada canal, por uma bomba de infusão de baixa complacência (JS Biomedicals, Ventura, CA, EUA).

Os pacientes foram estudados na posição supina após 12 horas de jejum. $\mathrm{O}$ cateter foi introduzido pela narina. $\mathrm{Na}$ avaliação das contrações utilizaram-se os quatro canais do cateter, distanciados de $5 \mathrm{~cm}$ entre si. Localizou-se a abertura do canal proximal $2 \mathrm{~cm}$ abaixo do esfíncter superior do esôfago (ESE) e as outras a $7 \mathrm{~cm}, 12 \mathrm{~cm}$ e $17 \mathrm{~cm}$ distal ao ESE. Nesta posição foram realizadas cinco deglutições de $5 \mathrm{~mL}$ de água, com intervalo mínimo de 20 segundos entre elas. Os pacientes eram orientados a realizar uma única deglutição e, caso houvesse deglutição dupla, tosse, náusea ou movimentação do paciente, ela era repetida. $\mathrm{O}$ marcador de evento para deglutição líquida foi utilizada para indicar cada deglutição.

Utilizando o programa Polygram Upper GI versão 6,4 (Gastrosoft, Stockholm, Suécia), mediram-se a amplitude, a duração, a área sob a curva (ASC) e o tempo de propagação das contrações, bem como o percentual de falhas de contração e de contrações peristálticas, simultâneas e não propagadas. A contração foi considerada falha quando, após a deglutição de água, não havia aumento de pressão no local onde ela era registrada. As contrações foram consideradas peristálticas quando o início do aumento de pressão entre dois locais consecutivos ocorreu com intervalo de um segundo ou mais, e consideradas simultâneas quando este intervalo foi inferior a um segundo. Contrações não propagadas foram aquelas interrompidas entre dois locais consecutivos do esôfago. Consideraram-se as medidas realizadas a $2 \mathrm{~cm}$ do ESE como sendo representativas do esôfago proximal, e aquelas realizadas a $17 \mathrm{~cm}$ como sendo esôfago distal ${ }^{(6)}$.

Para análise estatística, utilizou-se o teste de análise de variância (ANOVA), o teste de Tukey para múltiplas comparações e o teste de Fisher. Os resultados são apresentados como média, desvio padrão e percentagem.

\section{RESULTADOS}

Aos $12 \mathrm{~cm}$ e $17 \mathrm{~cm}$ do ESE a média da amplitude da contração dos pacientes com esofagite grave foi menor do que a amplitude dos pacientes sem esofagite ou com esofagite moderada $(P<0,05$, Tabela 1). Aos $2 \mathrm{~cm} \mathrm{e} 7 \mathrm{~cm}$ do ESE não houve diferenças entre os grupos.

TABELA 1 - Amplitude e duração das contrações esofágicas em pacientes com sintomas de doença do refluxo gastroesofágico sem esofagite (SE, $\mathrm{n}=42)$, com esofagite moderada $(\mathrm{EM}, \mathrm{n}=47)$ e com esofagite grave (EG, $\mathrm{n}=15)$, medidas a $2,7,12$ e $17 \mathrm{~cm}$ do esfíncter superior do esôfago, após a deglutição de água (média \pm DP)

\begin{tabular}{lcccc}
\hline \multicolumn{5}{c}{ Amplitude (mm Hg) } \\
\hline \multirow{5}{*}{ SE } & $2 \mathrm{~cm}$ & $7 \mathrm{~cm}$ & $12 \mathrm{~cm}$ & $17 \mathrm{~cm}$ \\
EM & $84,8 \pm 39,2$ & $42,0 \pm 25,4$ & $80,6 \pm 34,2^{*}$ & $94,5 \pm 32,9 * *$ \\
EG & $84,8 \pm 41,7$ & $39,1 \pm 15,0$ & $78,7 \pm 35,0^{*}$ & $93,5 \pm 45,3 * *$ \\
& $95,6 \pm 45,1$ & $36,1 \pm 27,5$ & $54,1 \pm 24,0$ & $55,6 \pm 26,8$ \\
\hline & $2 \mathrm{~cm}$ & Duração (segundos) & $17 \mathrm{~cm}$ \\
SE & $7 \mathrm{~cm}$ & $12 \mathrm{~cm}$ & $4,1 \pm 1,1$ \\
EM & $2,6 \pm 0,8$ & $3,0 \pm 0,7$ & $3,5 \pm 0,8$ & $3,9 \pm 0,9$ \\
EG & $2,7 \pm 0,8$ & $2,0 \pm 0,7$ & $3,5 \pm 0,7$ & $3,4 \pm 1,1$ \\
\hline$* P<0,05$ vs esofagite grave (EG) & $2,8 \pm 0,8$ & $3,3 \pm 0,9$ &
\end{tabular}

Aos $17 \mathrm{~cm}$ do ESE a área sob a curva das contrações foi menor nos pacientes com esofagite grave, quando comparada com os pacientes sem esofagite ou com esofagite moderada $(P<0,01$, Tabela 2), sem diferenças aos $2 \mathrm{~cm}$ e $7 \mathrm{~cm}$ do ESE.

TABELA 2 - Área sob a curva das contrações esofágicas e intervalo de tempo entre o início das contrações peristálticas em pacientes com sintomas de doença do refluxo gastroesofágico sem esofagite (SE, $\mathrm{n}=42)$, com esofagite moderada $(\mathrm{EM}, \mathrm{n}=47)$ e com esofagite grave (EG, $\mathrm{n}=15)$, medidas a 2, 7, 12 e $17 \mathrm{~cm}$ do esfíncter superior do esôfago, após a deglutição de água (média \pm DP)

\begin{tabular}{lcccc}
\hline \multicolumn{5}{c}{ Área sob a curva $(\mathrm{mm} \mathrm{Hg} \mathrm{x} \mathrm{s})$} \\
\hline & $2 \mathrm{~cm}$ & $7 \mathrm{~cm}$ & $12 \mathrm{~cm}$ & $17 \mathrm{~cm}$ \\
SE & $103,6 \pm 53,4$ & $76,5 \pm 62,7$ & $158,0 \pm 91,2^{*}$ & $214,3 \pm 114,0^{* *}$ \\
EM & $109,7 \pm 70,8$ & $67,5 \pm 29,7$ & $145,6 \pm 75,8$ & $192,1 \pm 118,7 * *$ \\
EG & $137,9 \pm 95,6$ & $63,2 \pm 52,5$ & $106,6 \pm 71,2$ & $111,1 \pm 55.5$ \\
\hline \multicolumn{5}{c}{ Intervalo de tempo (segundos) } \\
\hline \multirow{5}{*}{ SE } & $2 \rightarrow 7 \mathrm{~cm}$ & $7 \rightarrow 12 \mathrm{~cm}$ & $12 \rightarrow 17 \mathrm{~cm}$ \\
EM & $2,5 \pm 0,8$ & 1,2 & $1,8 \pm 0,5$ & $1,8 \pm 1,0$ \\
EG & $2,8 \pm 0,9$ & $1,8 \pm 0,6$ & $2,0 \pm 1,0$ \\
\hline
\end{tabular}

$P<0,05$ vs esofagite grave $(E G)$ $* * P<0,01$ vs esofagite grave $(E G)$ 
Não houve diferenças entre os grupos quanto à duração das contrações e ao intervalo de tempo entre o início das contrações (Tabelas $1,2)$. Também não houve diferenças entre os grupos em relação à percentagem de falhas de contração e percentagem de contrações peristálticas, simultâneas e não propagadas (Tabela 3 ).

Nos três grupos não houve diferença entre os pacientes com mais e aqueles com menos de 50 anos (Figuras 1,2).

\section{DISCUSSÃO}

Os resultados indicam que os pacientes com lesões mais intensas provocadas pelo refluxo gastroesofágico, têm menor amplitude das contrações esofágicas em parte distal do esôfago, do que pacientes com lesões menos intensas ou sem lesões, e que o fato de o paciente ser mais idoso não deve influenciar as contrações.

TABELA 3 - Percentagem de falhas de contração e de contrações esofágicas peristálticas, simultâneas ou não propagadas em pacientes com sintomas de doença do refluxo gastroesofágico sem esofagite (SE, $n=42)$, com esofagite moderada $(E M, n=47)$ e com esofagite grave (EG, $n=$ 15), medidas a 2, 7, 12 e $17 \mathrm{~cm}$ do esfíncter superior do esôfago, após a deglutição de água (média \pm DP)

\begin{tabular}{|c|c|c|c|c|c|c|c|}
\hline & \multicolumn{3}{|c|}{ Falhas } & \multicolumn{4}{|c|}{ Peristálticas } \\
\hline & $2 \mathrm{~cm}$ & $7 \mathrm{~cm}$ & $12 \mathrm{~cm}$ & $17 \mathrm{~cm}$ & $2 \rightarrow 7 \mathrm{~cm}$ & $7 \rightarrow 12 \mathrm{~cm}$ & $12 \rightarrow 17 \mathrm{~cm}$ \\
\hline SE & 0,5 & 17,1 & 8,6 & 11,0 & 76,7 & 67,6 & 71,4 \\
\hline EM & 0,9 & 15,3 & 3,8 & 2,6 & 77,9 & 67,2 & 81,7 \\
\hline \multirow[t]{3}{*}{ EG } & 0,0 & 18,7 & 8,0 & 9,3 & 73,3 & 61,3 & 73,3 \\
\hline & \multicolumn{3}{|c|}{ Simultâneas } & & \multicolumn{3}{|c|}{ Não propagadas } \\
\hline & $2 \rightarrow 7 \mathrm{~cm}$ & $7 \rightarrow 12 \mathrm{~cm}$ & $12 \rightarrow 17 \mathrm{~cm}$ & & $2 \rightarrow 7 \mathrm{~cm}$ & $7 \rightarrow 12 \mathrm{~cm}$ & $12 \rightarrow 17 \mathrm{~cm}$ \\
\hline SE & 2,9 & 10,5 & 12,4 & & 20,4 & 21,9 & 16,2 \\
\hline EM & 5,1 & 13,6 & 10,6 & & 17,0 & 19,2 & 7,7 \\
\hline EG & 6,7 & 17,3 & 17,3 & & 20,0 & 21,4 & 9,4 \\
\hline
\end{tabular}

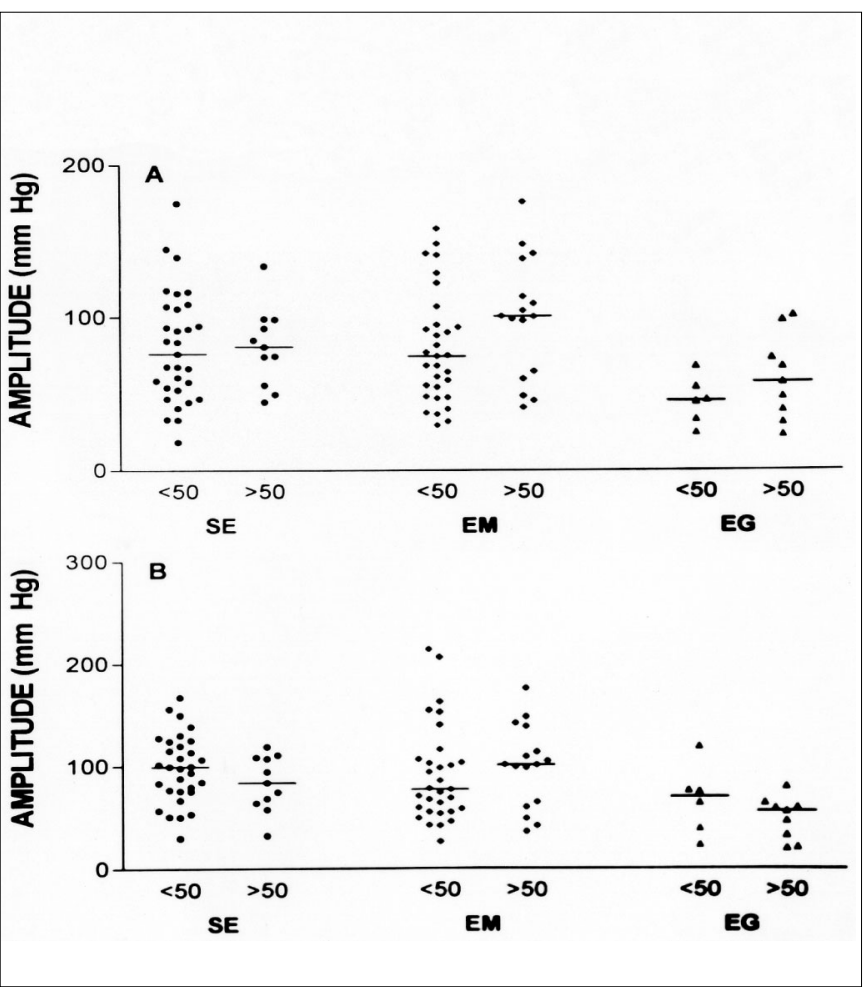

FIGURA 1 - Amplitude das contrações esofágicas medidas a $12 \mathrm{~cm}(\mathrm{~A})$ e $17 \mathrm{~cm}(\mathrm{~B})$ do esfíncter superior do esôfago em pacientes com sintomas de refluxo gastroesofágico sem esofagite (SE), com esofagite moderada (EM) e com esofagite grave (EG), com idades abaixo de 50 anos e acima de 50 anos. As linhas horizontais representam as medianas
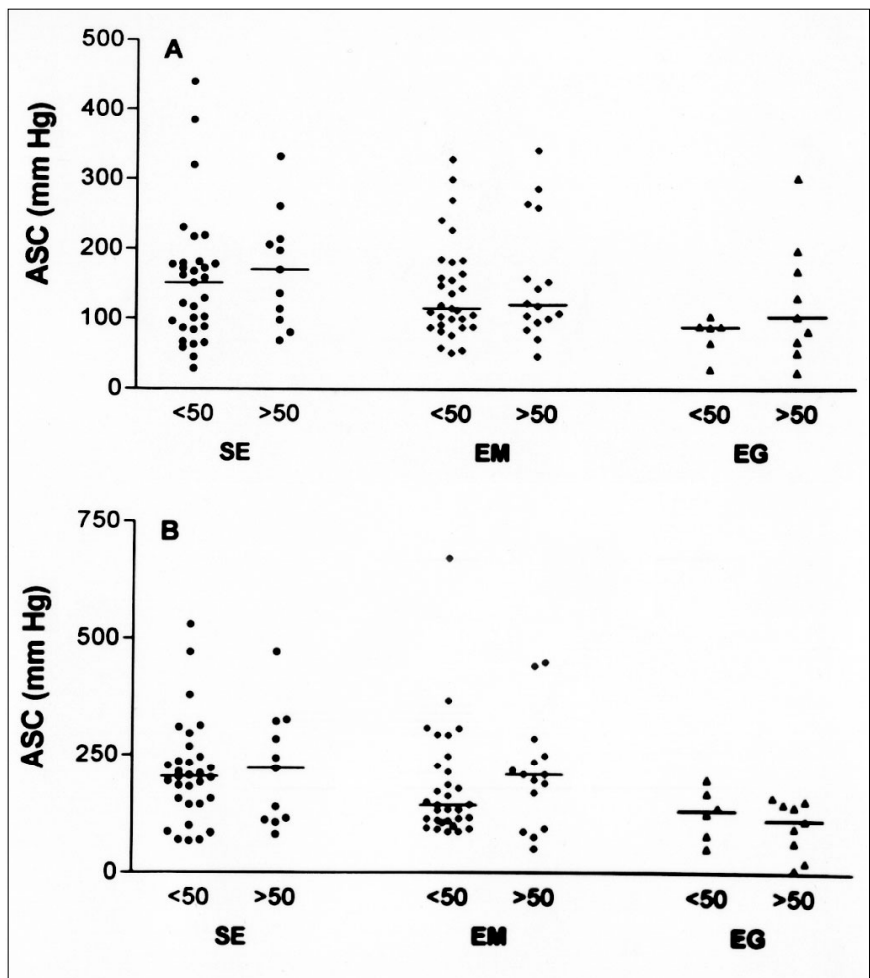

FIGURA 2 - Área sob a curva (ASC) das contrações esofágicas medidas a $12 \mathrm{~cm}(\mathrm{~A})$ e $17 \mathrm{~cm}(\mathrm{~B})$ do esfíncter superior do esôfago em pacientes com sintomas de refluxo gastroesofágico sem esofagite (SE), com esofagite moderada (EM) e com esofagite grave (EG), com idades abaixo de 50 anos e acima de 50 anos. As linhas horizontais representam as medianas 
A amplitude das contrações em parte distal do esôfago nos pacientes com esofagite grave, não diminuiu até valores que caracterizam a motilidade de esôfago ineficaz ${ }^{(15)}$, descrita como amplitude em esôfago distal inferior a $30 \mathrm{~mm} \mathrm{Hg}$ ou proporção de contrações não propagadas igual ou maior que $30 \%$ do total de deglutições líquidas. Entretanto, a amplitude das contrações observadas em parte distal do esôfago dos pacientes com esofagite grave foi inferior à dos pacientes sem lesões esofágicas ou com esofagite moderada, bem como inferior a de pessoas normais estudadas pelo método ${ }^{(3,5,8,20)}$.

Se pacientes com doença mais grave têm amplitude da contração diminuída em parte distal do esôfago, é importante entender o que isto significa para a evolução da doença. Pacientes com diminuição da amplitude presente na motilidade do esôfago ineficaz ( $<30 \mathrm{~mm} \mathrm{Hg}$ ) têm aumento do tempo total de exposição do esôfago ao pH inferior a 4, e dificuldade na depuração esofágica de ácido, quando comparados com pacientes com motilidade normal ${ }^{(15)}$. Outro estudo demonstrou que perda importante das contrações esofágicas provoca aumento da exposição do esôfago distal ao volume e pH ácido quando na posição supina, havendo depuração normal quando na posição ortostática ${ }^{(22)}$. Independente da presença ou não das contrações esofágicas em partes média e distal do esôfago e independente da posição, a chegada da saliva deglutida ao esôfago distal não é interrompida ou mais demorada ${ }^{(6)}$. Entretanto, quando as contrações esofágicas estão ausentes, a depuração da saliva na posição supina está prolongada $^{(6)}$. É sugerido que este maior tempo para a depuração aumenta a possibilidade de desenvolvimento de adenocarcinoma na transição esofagogástrica ${ }^{(18)}$. Adenocarcinomas ocorrem no local onde a saliva encontra o suco gástrico ácido. A mistura dos dois gera compostos nitrogenados potencialmente mutagênicos e carcinogênicos ${ }^{(18)}$.

Motilidade do esôfago ineficaz de grau moderado não afeta a depuração de volume ou química do ácido refluído ${ }^{(22)}$. Os pacientes com esofagite grave estudados não tinham diminuição tão expressiva das contrações em esôfago distal, portanto não é esperado haver grandes prejuízos na depuração. Mesmo com esta consideração, a maior intensidade da esofagite esteve associada com diminuição média da amplitude distal, sugerindo que esta possa ter importância na determinação da gravidade da esofagite. Entretanto, há resultados que não confirmam a associação entre motilidade do esôfago ineficaz e a presença de lesão esofágica ${ }^{(16)}$. No esôfago proximal não foram observadas diferenças entre os grupos, o que confirma resultados anteriores ${ }^{(4,14)}$.

Não se observou efeito do envelhecimento nas contrações deste grupo de pacientes. $\mathrm{O}$ envelhecimento afeta as contrações, compromete o trânsito pelo esôfago ${ }^{(8)}$ e diminui a amplitude da contração $^{(7)}$. Quando da ocorrência de refluxo gastroesofágico, o tempo de exposição ao material refluído é maior nas pessoas idosas do que nos jovens ${ }^{(9)}$. Estes estudos foram realizados em pacientes com média de idade maior do que as idades daqueles estudados nesta série, portanto é possível que pacientes com DRGE mais idosos tenham alterações de motilidade esofágica com maior freqüência do que pacientes mais jovens.
Em pessoas normais, o trânsito de bolo líquido de baixa ou alta viscosidade, em posição supina ou sentada, é mais longo em parte distal do esôfago, quando comparado com a parte proximal ${ }^{(25)}$. A amplitude da contração é maior em parte distal do esôfago do que em parte proximal ${ }^{(3,20,25)}$. Nos resultados deste estudo os pacientes sem lesão esofágica ou com esofagite moderada tiveram amplitude das contrações em parte distal ( $17 \mathrm{~cm}$ do ESE), em média um pouco superior à amplitude em parte proximal ( $2 \mathrm{~cm}$ do ESE), mas nos pacientes com esofagite grave a média de amplitude foi menor em parte distal, quando comparado com a parte proximal, o que poderia aumentar o naturalmente mais longo tempo de trânsito pelo esôfago distal. Entretanto, existe a possibilidade do trânsito em esôfago distal não depender da amplitude da contração ${ }^{(6,25)}$, o que faz com que a presente observação de baixa amplitude de contração em pacientes com esofagite grave, como já havia sido observado anteriormente ${ }^{(14)}$, seja analisada com reservas quanto à associação causa-efeito. Outros fatores podem estar envolvidos determinando tanto a diminuição da amplitude, quanto a gravidade da esofagite. $\mathrm{O}$ comprometimento da motilidade em região distal do esôfago e proximal do estômago poderia determinar a baixa amplitude da contração, o relaxamento transitório do esfíncter inferior do esôfago associado ao refluxo gastroesofágico, a baixa pressão deste esfíncter e as alterações na acomodação do estômago proximal ao volume ingerido ${ }^{(2,12)}$, alterações que, embora bastante estudadas nos dias de hoje, ainda não são completamente conhecidas quanto a sua fisiopatologia.

É possível que a diminuição da amplitude seja conseqüência da esofagite. Este fato é sugerido por estudos que mostram que a amplitude e a proporção de contrações peristálticas aumentam após o tratamento clínico da esofagite de refluxo ${ }^{(7,19)}$. Outros trabalhos, porém, não confirmam estes resultados ${ }^{(23,24)}$. Prevalece a idéia de que o comprometimento da motilidade do esôfago é irreversível ${ }^{(15)}$, o que coloca esses pacientes, em conseqüência à má depuração esofágica, em maior risco para complicações do refluxo gastroesofágico, como esôfago de Barrett e estenoses, indicando a necessidade de supressão ácida mais efetiva.

Durante o exame manométrico posicionou-se a abertura lateral mais proximal do cateter a $2 \mathrm{~cm}$ do ESE com o objetivo de se avaliar as contrações em esôfago proximal. O comprimento do corpo do esôfago varia de 18 a $25 \mathrm{~cm}^{(1,10)}$ e não tem relação com a altura ou peso do indivíduo ${ }^{(17)}$. Estudo realizado em um número grande de pacientes $(n=212)$ encontrou que o comprimento do corpo do esôfago, medido pelo método manométrico, tem média e desvio padrão de $22,9 \pm 2,9 \mathrm{~cm}$, mediana de $23,0 \mathrm{~cm}^{(17)}$. Tendo em consideração estes resultados, entende-se que as medidas realizadas a $17 \mathrm{~cm}$ do ESE eram representativas das contrações em metade distal do esôfago, a menos de $8 \mathrm{~cm}$ do esfíncter inferior do esôfago ${ }^{(6)}$, embora não se possa afirmar que foram todas realizadas à mesma distância do esfíncter.

Em conclusão, a amplitude das contrações esofágicas em parte distal do esôfago de pacientes com esofagite grave foram menores do que de pacientes com sintomas de refluxo, mas sem lesão esofágica, e de pacientes com esofagite moderada. Não se observou efeito da idade nas contrações esofágicas. 
Dantas RO, Aprile LRO. Aging and esophageal motility in patients with gastroesophageal reflux disease. Arq Gastroenterol 2006;43(2):107-11.

ABSTRACT - Background - Alterations of esophageal contractions may worsen the esophageal lesions caused by gastroesophageal reflux. The impairment of the contractions may be localized only in the distal esophagus or in the entire esophageal body, and may be worse with the aging process. Aims - To evaluate the proximal and distal esophageal contractions in patients with gastroesophageal reflux symptoms with or without esophagitis. Patients and Methods - We studied esophageal motility in 104 patients with gastroesophageal reflux symptoms, 42 with normal esophageal endoscopic examination, 47 with mild esophagitis and 15 with severe esophagitis. The esophageal contractions were measured by the manometric method at 2, 7, 12 and $17 \mathrm{~cm}$ from the upper esophageal sphincter, after five swallows of a $5 \mathrm{~mL}$ bolus of water. Results - The amplitude and area under the curve of contractions were lower in patients with severe esophagitis than in patients without esophagitis or with mild esophagitis in the distal part of the esophageal body (17 $\mathrm{cm}$ from the upper esophageal sphincter). In the proximal esophageal body there was no difference in amplitude or area under the curve. In the entire esophageal body there was no difference between the three groups of patients in duration, velocity of peristaltic contractions, or proportion of failed, simultaneous, nonpropagated or peristaltic contractions. There was no difference between the patients with less than 50 years or with more than 50 years of age. Conclusions - Patients with severe esophagitis had lower distal esophageal contraction amplitude than patients without esophagitis or with moderate esophagitis. There was no effect of aging on esophageal contractions.

HEADINGS - Gastroesophageal reflux. Esophagitis, peptic. Esophageal motility disorders. Aging.

\section{REFERÊNCIAS BIBLIOGRÁFICAS}

1. Biancani P, Harnett KM, Behar J. Esophageal motor function. In: Yamada T, editor Textbook of gastroenterology. $4^{\text {th }}$ edition. Philadelphia: Lippincott Williams \& Wilkins; 2003. v.1, p.166-94.

2. Cunningham KM, Horowitz M, Riddell PS, Maddem GI, Myers JC, Holloway RH, Wishart JM, Jamieson GG. Relations among autonomic nerve dysfunction, oesophageal motility, and gastric emptying in gastro-oesophageal reflux disease. Gut. 1991;32:1436-40

3. Dantas RO. Effect of successive swallows on oesophageal motility of normal volunteers, patients with Chagas' disease and patients with idiopathic achalasia. Neurogastroenterol Motil. 2003;15:57-62.

4. Dantas RO, Aprile LRO. Esophageal striated muscle contractions in patients with gastroesophageal reflux symptoms. Dig Dis Sci. 2002;47:2586-90.

5. Dantas RO, Deghaide NHS, Donadi EA. Esophageal motility of patients with Chagas disease and idiopathic achalasia. Dig Dis Sci. 2001;46:1200-6.

6. Dantas RO, Oliveira RB, Aprile LRO, Hara SH, Sifrim DA. Saliva transport to the distal esophagus. Scand J Gastroenterol. 2005;40:1010-6.

7. Deprez P, Fiasse R. Healing of severe esophagitis improves esophageal peristaltic dysfunction. Dig Dis Sci. 1999;44:125-33.

8. Ferriolli E, Dantas RO, Oliveira RB, Braga FJHN. The influence of ageing on oesophageal motility after ingestion of liquids with different viscosities. Eur J Gastroenterol Hepatol. 1996;8:793-8.

9. Ferriolli E, Oliveira RB, Matsuda NM, Braga FJHN, Dantas RO. Aging, esophageal motility, and gastroesophageal reflux. J Am Geriatr Soc. 1998;46:1534-7.

10. Goyal RK, Prasad M, Chang HY. Functional anatomy and physiology of swallowing and esophageal motility. In: Castell DO, Richter JE, editors. The esophagus. $4^{\text {th }}$ edition. Philadelphia: Lippincott Williams \& Wilkins; 2004. p.1-36.

11. Helm JF, Dodds WJ, Riedel DR, Teeter BC, Hogan WJ, Arndorfer RC. Determinants of esophageal acid clearance in normal subjects. Gastroenterology. 1983;85:607-12.

12. Herculano Jr JLR, Troncon LEA, Aprile LRO, Moraes ER, Secaf M, Onofre PHC, Dantas RO, Oliveira RB. Diminished retention of food in proximal stomach correlates with increased acidic reflux in patients with gastroesophageal reflux disease and dyspeptic symptoms. Dig Dis Sci. 2004;49:750-6.

13. Kahrilas PJ, Dodds WJ, Hogan WJ. Effect of peristaltic dysfunction on esophageal volume clearance. Gastroenterology. 1988;94:73-80.
14. Kahrilas PJ, Dodds WJ, Hogan WJ, Kern M, Arndorfer RC, Reece A. Esophageal peristaltic dysfunction in peptic esophagitis. Gastroenterology. 1986;91:897-904.

15. Leite LP, Johnston BT, Barrett J, Castell JA, Castell DO. Ineffective esophageal motility (IEM). The primary finding in patients with nonspecific esophageal motility disorder. Dig Dis Sci 1997;42:1859-65.

16. Lemme EMO, Abrahão Jr LJ, Manhães Y, Shechter R, Carvalho BB, Alvariz A. Ineffective esophageal motility in gastroesophageal erosive reflux disease and in nonerosive reflux disease. Are they different? J Clin Gastroenterol. 2005;39:224-7.

17. Li Q, Castell JA, Castell DO. Manometric determination of esophageal length. Am J Gastroenterol. 1994;89:722-5.

18. McColl KEL. When saliva meets acid: chemical warfare at the oesophagogastric junction. Gut. 2005;54:1-3.

19. Meshkinpour H. Esophageal aperistalsis and gastroesophageal reflux disorder: return of peristalsis after H2-blocher therapy. Am J Gastroenterol. 1995;90:910-4.

20. Miranda ALM, Dantas RO. Esophageal contractions and oropharyngeal and esophageal transits in patients with iron deficiency anemia. Am J Gastroenterol. 2003;98:1000-4.

21. Savary M, Miller G. The esophagus. In: Handbook and atlas of endoscopy. Solothurn, Switzerland: Gassman; 1978. p.135-42.

22. Simrém M, Silny J, Holloway R, Tack J, Janssens J, Sifrim D. Relevance of ineffective oesophageal motility during oesophageal acid clearance. Gut. 2003;52:784-90.

23. Timmer R, Breumelhof R, Nadorp JH, Smout AJ. Oesophageal motility and gastrooesophageal reflux before and after healing of reflux oesophagitis. A study using 24 hour ambulatory $\mathrm{pH}$ and pressure monitoring. Gut. 1994;35:1519-22.

24. Williams D, Thompson DG, Heggie L, O'Hanrahan T, Bancewicz J. Esophageal clearance function following treatment of esophagitis. Gastroenterology. 1994;106:108-16.

25. Wise JL, Murray JA, Conklin JL. Regional differences in oesophageal motor function. Neurogastroenterol Motil. 2004;16:31-7. 